\title{
ON THE PROPERTIES OF SOLUTION OF STOCHASTIC DIFFERENTIAL EQUATION WITH RESPECT TO INITIAL DATA IN ONE-DIMENSIONAL CASE
}

\author{
FATIMA BENZIADI
}

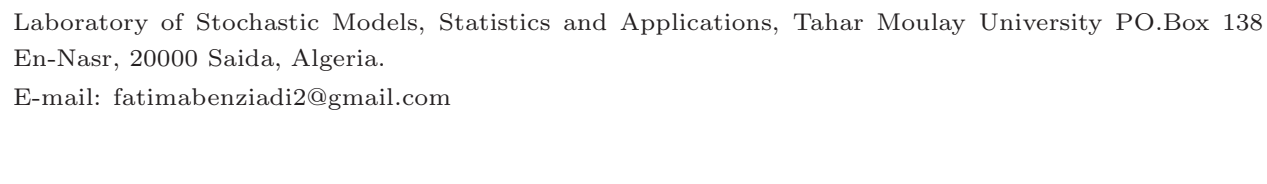

Abstract

The one-default models are widely applied in modeling financial risk and in price valuation of financial products such as Credit default swap. In this paper, we are interested essentially to the so-called natural model. This model is expressed by a stochastic differential equation called $\downarrow$-equation, this equation displays the evolution of the defaultable market. So, on the same model and with some assumptions, we will study the property of homeomorphism of the stochastic flow generated by the natural model in a one-dimensional case and with some modifications, based on an important theory of Hiroshi Kunita. This is the main motivation of our research.

\section{Introduction}

The notion of the stochastic flow associated with a stochastic differential equation has been studied by several authors, e.g., Elworthy [3], Malliavin [4], Ikeda-Watanabe [5], Bismut [6]. In this work, we are interested by the stochastic flow generated by the so-called $\downarrow$-model, it is one-default model which gives the conditional law of a random time with respect to a reference filtration. This models are widely applied in modeling financial risk and price valuation of financial products.

Precisely, it is proved in [1] that, for any continuous local martingale $Y$, for any Lipschitz function $f$ on $\mathbb{R}$ null at the origin, there exist a probability

Received March 30, 2020 and in revised form June 15, 2020.

AMS Subject Classification: 60G17, 60H05.

Key words and phrases: Credit risk, stochastic flow, stochastic differential geometry, diffeomorphism. 
measure $\mathbb{Q}$ and a random time $\tau>0$ on an extension of $(\Omega, \mathbb{F}, \mathbb{P})$, such that the survival probability of $\tau$, i.e., $\mathbb{Q}\left[\tau>t \mid \mathcal{F}_{t}\right]$ is equal to $Z_{t}$ for $t \geq 0$. In the same last reference, it is also shown that there exist several solutions and that an increasing family of martingales, combined with a stochastic differential equation, constitutes a natural way to construct these solutions, which means that $X_{t}^{u}=\mathbb{Q}\left[\tau \leq \mid \mathcal{F}_{t}\right], 0<u, t<\infty$, satisfy the following stochastic differential equation :

$$
\left(\natural_{u}\right):\left\{\begin{array}{l}
d X_{t}=X_{t}\left(-\frac{e^{-\Lambda_{t}}}{1-Z_{t}} d N_{t}+f\left(X_{t}-\left(1-Z_{t}\right)\right) d Y_{t}\right), \quad t \in[u, \infty) \\
X_{u}=x
\end{array}\right.
$$

where the initial condition $x$ can be any $\mathcal{F}_{u}$-mesurable random variable.

The main result of this paper is to prove the homeomorphism property of the stochastic flow generated by the stochastic flow associated with the q-equation based on Hiroshi Kunita theory, but we impose the following hypotheses:

Hypothesis 1. We keep the same naturel model, but we assume that all the processes indicated in the -equation take real values. Thus, we impose that the coefficients of this equation are Lipschitz continuous.

Hypothesis 2. We always assume the hypothesis mentioned in [1], which denoted that the stochastic integral $\int_{u}^{t} \frac{e^{-\Lambda_{s}}}{1-Z_{s}} d N_{s}, u \leq t<\infty$, exists and defines a local martingale.

Remark 1. With these assumptions, we recall that the solution of the $\downarrow$ equation is continuous according to the article [7].

Remark 2. It is reported here that the H. Kunita theory appearing in Section 2 was done for multidimensional processes. Therefore, to obtain our result in the one-default model, it suffices to apply the unidimensional version of the Itô's formula.

\section{The Stochastic Flow of Stochastic Differential Equation}

This section is borrowed from [2]. 
Let $G_{1}(x), \ldots, G_{r}(x)$ be continuous mappings from $\mathbb{R}^{d}$ into itself and $M_{t}^{1}, \ldots, M_{t}^{r}$ be continuous semimartingales defined on a probability space $\left(\Omega, \mathbb{F}, \mathbb{P} ; \mathbb{F}_{t}\right)$. Here $\mathbb{F}_{t}, 0 \leq t<\infty$ is an increasing family of sub $\sigma$-fields of $\mathbb{F}$ such that $\wedge_{\varepsilon>0} \mathbb{F}_{t+\varepsilon}=\mathbb{F}_{t}$ holds for each $t$. Consider an Itô stochastic differential equation (SDE) on $\mathbb{R}^{d}$;

$$
d \xi_{t}=\sum_{j=1}^{r} G_{j}\left(\xi_{t}\right) d M_{t}^{j}
$$

A sample continuous $\mathbb{F}_{t}$-adapted stochastic process $\xi_{t}$ with values in $\mathbb{R}^{d}$ is called a solution of (2.1), if it satisfies

$$
\xi_{t}=\xi_{0}+\sum_{j=1}^{d} \int_{0}^{t} G_{j}\left(\xi_{s}\right) d M_{s}^{j}
$$

where the right hand side is the Ito integral.

Concerning coefficients of the equation, we will assume in this section that they are Lipschitz continuous, i.e., there is a positive constant $L$ such that

$$
\left|G_{j}^{i}(x)-G_{j}^{i}(y)\right| \leq L|x-y|, \quad \forall x, y \in \mathbb{R}^{d}
$$

holds for all indices $i, j$, where $G_{j}^{i}(x)$ is the $i$-th component of the vector function $G_{j}(x)$. Then for a given point $x$ of $\mathbb{R}^{d}$, the equation has a unique solution such that $\xi_{0}=0$. We denote it as $\xi_{t}(x)$ or $\xi_{t}(x, \omega)$. It is continuous in $(t, x)$ a.s. In fact, the following proposition is well known.

Proposition $1([8]) . \xi_{t}(x, \omega)$ is continuous in $[0, \infty) \times \mathbb{R}^{d}$ for almost all $\omega$. Furthermore, for any $T>0$ and $p \geq 2$, there is a positive constant $K_{p, T}^{(1)}$ such that

$$
\mathbb{E}\left|\xi_{t}(x)-\xi_{s}(y)\right|^{p} \leq K_{p, T}^{(1)}\left(|x-y|^{p}+|t-s|^{\frac{p}{2}}\right)
$$

holds for all $x, y$ of $\mathbb{R}^{d}$ and $t, s$ of $[0, T]$.

We thus regard that for fixed $t, \xi_{t}(\cdot, \omega)$ is a continuous map from $\mathbb{R}^{d}$ into itself for almost all $\omega$. The purpose of this section is to prove that map $\xi_{t}(\cdot, \omega)$ is one to one and onto, and that the inverse map $\xi_{t}^{-1}(\cdot, \omega)$ is also continuous. 
Theorem 1 ([2]). Suppose that $G_{1}, \ldots, G_{r}$ of equation (2.1) are Lipschitz continuous. Then the solution map $\xi_{t}(\cdot, \omega)$ is a homeomorphism of $\mathbb{R}^{d}$ for all t, a.s. $\omega$.

Remark 3. In case of one dimensional SDE, Ogura and Yamada 9] has shown the same result under a weaker condition, using a strong comparison theorem of solutions. In fact, if coefficients are Lipschitz continuous on any finite interval (local Lipschitzan) and if they are of linear growth, i.e., $\left|G_{j}(x)\right| \leq C(1+|x|)$ holds for all $x$ with some positive $C$, then the solution $\xi_{t}(\cdot, \omega)$ is homeomorphism for any $t$ a.s.

Remark 4. The (local) Lipschitz continuity of coefficients is crucial for the theorem. Ogura and Yamada [9] has given an example of one dimensional SDE with $\alpha$-Hölder continuous coefficients $\left(\frac{1}{2}<\alpha<1\right)$, which has a unique strong solution but does not have the "one to one" property.

Remark 5. It is enough to prove the theorem in case that $M_{t}^{i}, i=1, \ldots, r$ satisfy properties below: Let $M_{t}^{j}=B_{t}^{j}+A_{t}^{j}$ be the decomposition of semimartingale such that $B_{t}^{j}$ is a continuous local martingale and $A_{t}^{j}$ is a continuous process of bounded variation. Let $\left\langle B^{j}>_{t}\right.$ be the quadratic variation of $B_{t}^{j}$. Then it holds for each $j$ and $\forall s<t$,

$$
A_{t}^{j}-A_{s}^{j} \leq t-s,<B^{j}>_{t}-<B^{j}>_{s} \leq t-s, \quad \forall s<t .
$$

In the following discussion, condition (2.4) is always assumed. We will first show the "one to one" property. Our approach is based on several elementary inequalities.

Lemma 1 ([2]). Let $T>0$ and $p$ be any real number. Then there is a positive constant $K_{p, T}^{(2)}$ such that $\forall x, y \in \mathbb{R}^{d}$ and $\forall t \in[0, T]$,

$$
\mathbb{E}\left|\xi_{t}(x)-\xi_{s}(y)\right|^{p} \leq K_{p, T}^{(2)}|x-y|^{p}, \quad \forall x, y \in \mathbb{R}^{d}, \quad \forall t \in[0, T] .
$$

The above lemma shows that if $x \neq y$ then $\xi_{t}(x) \neq \xi_{t}(y)$ holds for all $t$ a.s. But it does not conclude that $\xi_{t}(\cdot, \omega)$ is "one to one", since the exceptional null set $N_{x, y}=\left\{\omega \mid \xi_{t}(x)=\xi_{t}(y)\right.$ for some $\left.t\right\}$ depends on the pair $(x, y)$. To overcome this point, we shall prove the following lemma. 
Lemma $2([8])$. Set

$$
\eta(x, y)=\frac{1}{\left|\xi_{t}(x)-\xi_{t}(y)\right|} .
$$

Then $\eta_{t}(x, y)$ is continuous in $[0, \infty) \times\left\{(x, y) \in \mathbb{R}^{2 d} \mid x \neq y\right\}$.

The above lemma leads immediately to the "one to one" property of the $\operatorname{map} \xi_{t}(\cdot, \omega)$ for all $t$ a.s. We shall next consider the onto property. We first establish

Lemma 3 ([2]). Let $T>0$ and $p$ be any real number. Then there is a positive constant $K_{p, T}^{(3)}$ such that

$$
\mathbb{E}\left(1+\left|\xi_{t}(x)\right|^{2}\right)^{p} \leq K_{p, T}^{(3)}\left(1+|x|^{2}\right)^{p}, \quad \forall x \in \mathbb{R}^{d}, \forall t \in[0, T]
$$

Remark 6. It holds $\left(1+|x|^{2}\right) \leq(1+|x|)^{2} \leq 2\left(1+|x|^{2}\right)$. Therefore, inequality (2.7) implies

$$
\mathbb{E}\left(1+\left|\xi_{t}(x)\right|\right)^{2 p} \leq 2^{|p|} K_{p, T}^{(3)}(1+|x|)^{2 p}
$$

Now taking negative $p$ in the above lemma, we see that $\left|\xi_{t}(x)\right|$ tends to infinity in probability as $x$ tends sequentially to infinity. We shall prove a stronger convergence. We claim

Lemma 4 ([2]). Let $\overline{\mathbb{R}^{d}}=\mathbb{R}^{d} \cup\{\infty\}$ be the one point compactification of $\mathbb{R}^{d}$. Set

$$
\eta_{t}(x)= \begin{cases}\frac{1}{1+\left|\xi_{t}(x)\right|}, & \text { if } x \in \mathbb{R}^{d} \\ 0, & \text { if } x=\infty\end{cases}
$$

Then $\eta_{t}(x, \omega)$ is a continuous map from $[0, \infty) \times \overline{\mathbb{R}^{d}}$ into $\mathbb{R}$ a.s.

Lemma $5([2])$. Define a stochastic process $\bar{\xi}_{t}$ on $\overline{\mathbb{R}^{d}}=\mathbb{R}^{d} \cup\{\infty\}$ by

$$
\bar{\xi}_{t}(x)= \begin{cases}\xi_{t}(x), & \text { if } x \in \mathbb{R}^{d} \\ \infty, & \text { if } x=\infty\end{cases}
$$

Then $\bar{\xi}_{t}(x)$ is continuous in $[0, \infty) \times \overline{\mathbb{R}^{d}}$.

Now the map $\bar{\xi}_{t}$ is a homeomorphism of $\overline{\mathbb{R}^{d}}$, since it is one to one, onto and continuous. Since $\infty$ is the invariant point of the map $\bar{\xi}_{t}$, we see that $\xi_{t}$ 
is a homeomorphism of $\mathbb{R}^{d}$. This completes the proof of Theorem 1

\section{Main Result}

In our model and with the assumptions set out in Section 1, we show the homeomorphic property of the solution of the t-equation by applying the lemmas introduced by H.Kunita presented in the previous section. We take $\varepsilon=p$ and $\beta=p-n$ with $p>0$, we have for $u \leq s \leq t$ :

$$
X_{t}^{u}(x)=x+\int_{u}^{t} X_{s}\left(-\frac{e^{-\Lambda_{s}}}{1-Z_{s}}\right) d N_{s}+\int_{u}^{t} X_{s} f\left(X_{s}-\left(1-Z_{s}\right)\right) d Y_{s} .
$$

We know that the quantity $f\left(X_{s}-\left(1-Z_{s}\right)\right)$ is bounded because $f$ is a Lipschitz function, but as we do not know a priori if the quantity $\left(-\frac{e^{-\Lambda_{s}}}{1-Z_{s}}\right)$ is finite or not, we introduce the stopping time $\tau_{n}=\inf \left\{t, 1-Z_{t}<\frac{1}{n}\right\}$. Therefore, we assume the process $\tilde{X}$ instead of X: $d \tilde{X}_{t}=\tilde{X}_{t}\left(-\frac{e^{-\Lambda_{t}}}{1-Z_{t \wedge \tau_{n}}} d N_{t}+\right.$ $\left.f\left(\tilde{X}_{t}-\left(1-Z_{t}\right)\right) d Y_{t}\right)$. Such as $\tilde{X}_{t}=X_{t}, \quad \forall t \leq \tau_{n}, n \in \mathbb{N}$.

\subsection{Proof of the one to one property}

In this part we will apply the Lemma 1 to the one-default model. So if $x=y$ the inequality is clearly satisfied for any constant $\tilde{K}_{p, T}^{2}$. We shall assume $x=y$. Let $\tilde{\varepsilon}$ be an arbitrary positive number and:

$$
\sigma_{\tilde{\varepsilon}}=\inf \left\{t>0,\left|\tilde{X}_{t}^{u}(x)-\tilde{X}_{t}^{u}(y)\right|<\tilde{\varepsilon}\right\}
$$

denote $A_{t}=\tilde{X}_{t}^{u}(x)-\tilde{X}_{t}^{u}(y)$, and we shall apply Itô's formula to the function $f(z)=|z|^{p}$. Then it holds for $t<\tilde{\varepsilon}$;

$$
\begin{aligned}
& \tilde{X}_{t}^{u}(x)=x+\int_{u}^{t} \tilde{X}_{s}\left(-\frac{e^{-\Lambda_{s}}}{1-Z_{s \wedge \tau_{n}}}\right) d N_{s}+\int_{u}^{t} \tilde{X}_{s} f\left(\tilde{X}_{s}-\left(1-Z_{s}\right)\right) d Y_{s} \\
& d \tilde{X}_{t}^{u}(x)=\tilde{X}_{t}\left(-\frac{e^{-\Lambda_{t}}}{1-Z_{t \wedge \tau_{n}}} d N_{t}+f\left(\tilde{X}_{t}-\left(1-Z_{t}\right)\right) d Y_{t}\right) \\
& \left|\tilde{X}_{t}^{u}(x)-\tilde{X}_{t}^{u}(y)\right|^{p}-|x-y|^{p} \\
& =\int_{u}^{t} \frac{\partial f}{\partial z}\left(\tilde{X}_{t}^{u}(x)-\tilde{X}_{t}^{u}(y)\right)
\end{aligned}
$$




$$
\begin{aligned}
& \times\left[\tilde{X}_{s}(x)\left(-\frac{e^{-\Lambda_{s}}}{1-Z_{s \wedge \tau_{n}}}\right) d N_{s}+\tilde{X}_{s}(x) f\left(\tilde{X}_{s}(x)-\left(1-Z_{s}\right)\right) d Y_{s}\right. \\
& \left.-\tilde{X}_{s}(y)\left(-\frac{e^{-\Lambda_{s}}}{1-Z_{s \wedge \tau_{n}}}\right) d N_{s}+\tilde{X}_{s}(y) f\left(\tilde{X}_{s}(y)-\left(1-Z_{s}\right)\right) d Y_{s}\right] \\
& +\frac{1}{2} \int_{u}^{t} \frac{\partial^{2} f}{\partial z^{2}}\left(\tilde{X}_{s}^{u}(x)-\tilde{X}_{s}^{u}(y)\right) \\
& \times\left[\tilde{X}_{s}(x)\left(-\frac{e^{-\Lambda_{s}}}{1-Z_{s \wedge \tau_{n}}}\right) d N_{s}+\tilde{X}_{s}(x) f\left(\tilde{X}_{s}(x)-\left(1-Z_{s}\right)\right) d Y_{s}\right. \\
& \left.-\tilde{X}_{s}(y)\left(-\frac{e^{-\Lambda_{s}}}{1-Z_{s \wedge \tau_{n}}}\right) d N_{s}+\tilde{X}_{s}(y) f\left(\tilde{X}_{s}(y)-\left(1-Z_{s}\right)\right) d Y_{s}\right]^{2} \\
\mid & \tilde{X}_{t}^{u}(x)-\left.\tilde{X}_{t}^{u}(y)\right|^{p}-|x-y|^{p} \\
= & \int_{u}^{t} \frac{\partial f}{\partial z}\left(\tilde{X}_{t}^{u}(x)-\tilde{X}_{t}^{u}(y)\right) \times\left[\left(\tilde{X}_{s}(x)-\tilde{X}_{s}(y)\right)\left(-\frac{e^{-\Lambda_{s}}}{1-Z_{s \wedge \tau_{n}}}\right) d N_{s}\right. \\
& \left.+\left(\tilde{X}_{s}(x) f\left(\tilde{X}_{s}(x)-\left(1-Z_{s}\right)\right)-\tilde{X}_{s}(y) f\left(\tilde{X}_{s}(y)-\left(1-Z_{s}\right)\right)\right) d Y_{s}\right] \\
& +\frac{1}{2} \int_{u}^{t} \frac{\partial^{2} f}{\partial z^{2}}\left(\tilde{X}_{s}^{u}(x)-\tilde{X}_{s}^{u}(y)\right) \times\left[\left(\tilde{X}_{s}(x)-\tilde{X}_{s}(y)\right)\left(-\frac{\Lambda_{s}}{1-Z_{s \wedge \tau_{n}}}\right) d N_{s}\right. \\
& \left.+\left(\tilde{X}_{s}(x) f\left(\tilde{X}_{s}(x)-\left(1-Z_{s}\right)\right)-\tilde{X}_{s}(y) f\left(\tilde{X}_{s}(y)-\left(1-Z_{s}\right)\right)\right) d Y_{s}\right]^{2} \\
\mid & \tilde{X}_{t}^{u}(x)-\left.\tilde{X}_{t}^{u}(y)\right|^{p}-|x-y|^{p}=\tilde{I}_{t}+\tilde{J}_{t}
\end{aligned}
$$

we start with $\tilde{I}_{t}$ :

$$
\begin{aligned}
\tilde{I}_{t}= & \int_{u}^{t} \frac{\partial f}{\partial z}\left(\tilde{X}_{t}^{u}(x)-\tilde{X}_{t}^{u}(y)\right) \times\left[\left(\tilde{X}_{s}(x)-\tilde{X}_{s}(y)\right)\left(-\frac{e^{-\Lambda_{s}}}{1-Z_{s \wedge \tau_{n}}}\right) d N_{s}\right. \\
& \left.+\left(\tilde{X}_{s}(x) f\left(\tilde{X}_{s}(x)-\left(1-Z_{s}\right)\right)-\tilde{X}_{s}(y) f\left(\tilde{X}_{s}(y)-\left(1-Z_{s}\right)\right)\right) d Y_{s}\right] .
\end{aligned}
$$

Noting

$$
\begin{aligned}
& \tilde{V}\left(\tilde{X}_{s}^{x}\right)=\tilde{X}_{s}(x) f\left(\tilde{X}_{s}(x)-\left(1-Z_{s}\right)\right) \\
& \tilde{V}\left(\tilde{X}_{s}^{y}\right)=\tilde{X}_{s}(y) f\left(\tilde{X}_{s}(y)-\left(1-Z_{s}\right)\right)
\end{aligned}
$$

such that

$$
\left|\tilde{V}\left(\tilde{X}_{s}^{x}\right)-\tilde{V}\left(\tilde{X}_{s}^{y}\right)\right| \leq \tilde{L}\left|\tilde{X}_{s}^{x}-\tilde{X}_{s}^{y}\right|
$$


and

$$
\frac{\partial f}{\partial z}=p|z|^{p-1}
$$

we put

$$
\tilde{I}_{t}=\tilde{I}_{t}^{1}+\tilde{I}_{t}^{2}
$$

such that

$$
\begin{aligned}
& \tilde{I}_{t}^{1}=\int_{u}^{t} \frac{\partial f}{\partial z}\left(\tilde{X}_{t}^{u}(x)-\tilde{X}_{t}^{u}(y)\right)\left(\tilde{X}_{s}(x)-\tilde{X}_{s}(y)\right)\left(-\frac{e^{-\Lambda_{s}}}{1-Z_{s \wedge \tau_{n}}}\right) d N_{s} \\
& \tilde{I}_{t}^{2}=\int_{u}^{t} \frac{\partial f}{\partial z}\left(\tilde{X}_{t}^{u}(x)-\tilde{X}_{t}^{u}(y)\right)\left(\tilde{V}\left(\tilde{X}_{s}^{x}\right)-\tilde{V}\left(\tilde{X}_{s}^{y}\right)\right) d Y_{s} .
\end{aligned}
$$

For $\tilde{I}_{t}^{1}$, we have:

$$
\begin{aligned}
\left|\frac{\partial f}{\partial z}\left(\tilde{X}_{t}^{u}(x)-\tilde{X}_{t}^{u}(y)\right)\left(\tilde{X}_{s}(x)-\tilde{X}_{s}(y)\right)\right| & \leq|p||z|^{p-1}\left|\tilde{X}_{s}(x)-\tilde{X}_{s}(y)\right| \\
& \leq|p|\left|\tilde{X}_{s}(x)-\tilde{X}_{s}(y)\right|^{p} .
\end{aligned}
$$

Therefore

$$
\tilde{I}_{t}^{1} \leq|p| \int_{u}^{t}\left|\tilde{X}_{s}(x)-\tilde{X}_{s}(y)\right|^{p} d s \times \int_{u}^{t}-\frac{e^{-\Lambda_{s}}}{1-Z_{s \wedge \tau_{n}}} d N_{s} .
$$

\section{Noting}

$Q_{t}=\int_{u}^{t}-\frac{e^{-\Lambda_{s}}}{1-Z_{s \wedge \tau_{n}}} d N_{s}$, it is a local martingale according to hypothesis 2 (so called the hypothesis $H_{Y}(C)[1]$ ). So

$$
\tilde{I}_{t}^{1} \leq|p| Q_{t} \int_{u}^{t}\left|\tilde{X}_{s}(x)-\tilde{X}_{s}(y)\right|^{p} d s
$$

For $\tilde{I}_{t}^{2}$, we have:

$$
\begin{aligned}
\left|\frac{\partial f}{\partial z}\left(\tilde{X}_{t}^{u}(x)-\tilde{X}_{t}^{u}(y)\right)\left(\tilde{V}\left(\tilde{X}_{s}^{x}\right)-\tilde{V}\left(\tilde{X}_{s}^{y}\right)\right)\right| & \leq|p||z|^{p-1} \tilde{L}\left|\tilde{X}_{s}(x)-\tilde{X}_{s}(y)\right| \\
& \leq|p| \tilde{L}\left|\tilde{X}_{s}(x)-\tilde{X}_{s}(y)\right|^{p} .
\end{aligned}
$$


Therefore

$$
\tilde{I}_{t}^{2} \leq|p| \tilde{L} \int_{u}^{t}\left|\tilde{X}_{s}(x)-\tilde{X}_{s}(y)\right|^{p} d s .
$$

So, we have

$$
\begin{aligned}
\tilde{I}_{t}=\tilde{I}_{t}^{1}+\tilde{I}_{t}^{2} & \leq|p| Q_{t} \int_{u}^{t}\left|\tilde{X}_{s}(x)-\tilde{X}_{s}(y)\right|^{p} d s+|p| \tilde{L} \int_{u}^{t}\left|\tilde{X}_{s}(x)-\tilde{X}_{s}(y)\right|^{p} d s \\
& \leq|p| \int_{u}^{t}\left|\tilde{X}_{s}(x)-\tilde{X}_{s}(y)\right|^{p} d s\left(Q_{t}+\tilde{L}\right) .
\end{aligned}
$$

Therefore, we have

$$
\left|\mathbb{E} \tilde{I}_{t \wedge \sigma_{\tilde{\varepsilon}}}\right| \leq|p|\left(Q_{t \wedge \sigma_{\tilde{\varepsilon}}}+\tilde{L}\right) \int_{u}^{t} \mathbb{E}\left|\tilde{X}_{s \wedge \sigma_{\tilde{\varepsilon}}}(x)-\tilde{X}_{s \wedge \sigma_{\tilde{\varepsilon}}}(y)\right|^{p} d s .
$$

Next,

$$
\begin{aligned}
\tilde{J}_{t}= & \frac{1}{2} \int_{u}^{t} \frac{\partial^{2} f}{\partial z^{2}}\left(\tilde{X}_{s}^{u}(x)-\tilde{X}_{s}^{u}(y)\right) \\
& \times\left[\left(\tilde{X}_{s}(x)-\tilde{X}_{s}(y)\right)\left(-\frac{e^{-\Lambda_{s}}}{1-Z_{s \wedge \tau_{n}}}\right) d N_{s}+\left(\tilde{V}\left(\tilde{X}_{s}^{x}\right)-\tilde{V}\left(\tilde{X}_{s}^{y}\right)\right) d Y_{s}\right]^{2} \\
\tilde{J}_{t}= & \frac{1}{2} \int_{u}^{t} \frac{\partial^{2} f}{\partial z^{2}}\left(\tilde{X}_{s}^{u}(x)-\tilde{X}_{s}^{u}(y)\right) \\
\times & {\left[\left(\tilde{X}_{s}(x)-\tilde{X}_{s}(y)\right)^{2}\left(-\frac{e^{-\Lambda_{s}}}{1-Z_{s \wedge \tau_{n}}}\right)^{2} d N_{s} d N_{s}+\left(\tilde{V}\left(\tilde{X}_{s}^{x}\right)-\tilde{V}\left(\tilde{X}_{s}^{y}\right)\right)^{2} d Y_{s} d Y_{s}\right.} \\
& \left.+2\left(\tilde{X}_{s}(x)-\tilde{X}_{s}(y)\right)\left(-\frac{e^{-\Lambda_{s}}}{1-Z_{s \wedge \tau_{n}}}\right)\left(\tilde{V}\left(\tilde{X}_{s}^{x}\right)-\tilde{V}\left(\tilde{X}_{s}^{y}\right)\right) d N_{s} d Y_{s}\right] .
\end{aligned}
$$

Noting $\tilde{J}_{t}=\frac{1}{2}\left[\tilde{J}_{t}^{1}+\tilde{J}_{t}^{2}+\tilde{J}_{t}^{3}\right]$ such that:

$$
\begin{aligned}
\tilde{J}_{t}^{1}= & \int_{u}^{t} \frac{\partial^{2} f}{\partial z^{2}}\left(\tilde{X}_{s}^{u}(x)-\tilde{X}_{s}^{u}(y)\right) \times\left(\tilde{X}_{s}(x)-\tilde{X}_{s}(y)\right)^{2}\left(-\frac{e^{-\Lambda_{s}}}{1-Z_{s \wedge \tau_{n}}}\right)^{2} d N_{s} d N_{s} \\
\tilde{J}_{t}^{2}= & \int_{u}^{t} \frac{\partial^{2} f}{\partial z^{2}}\left(\tilde{X}_{s}^{u}(x)-\tilde{X}_{s}^{u}(y)\right) \times\left(\tilde{V}\left(\tilde{X}_{s}^{x}\right)-\tilde{V}\left(\tilde{X}_{s}^{y}\right)\right)^{2} d Y_{s} d Y_{s} \\
\tilde{J}_{t}^{3}= & 2 \int_{u}^{t} \frac{\partial^{2} f}{\partial z^{2}}\left(\tilde{X}_{s}^{u}(x)-\tilde{X}_{s}^{u}(y)\right) \times\left(\tilde{X}_{s}(x)-\tilde{X}_{s}(y)\right)\left(-\frac{e^{-\Lambda_{s}}}{1-Z_{s \wedge \tau_{n}}}\right) \\
& \times\left(\tilde{V}\left(\tilde{X}_{s}^{x}\right)-\tilde{V}\left(\tilde{X}_{s}^{y}\right)\right) d N_{s} d Y_{s}
\end{aligned}
$$


and note that

$$
\frac{\partial^{2} f}{\partial z^{2}}=p(p-1)|z|^{p-2}
$$

For $\tilde{J}_{t}^{1}$ we have

$$
\begin{aligned}
& \left|\frac{\partial^{2} f}{\partial z^{2}}\left(\tilde{X}_{s}^{u}(x)-\tilde{X}_{s}^{u}(y)\right) \times\left(\tilde{X}_{s}(x)-\tilde{X}_{s}(y)\right)^{2}\right| \\
& \quad \leq\left.|p(p-1)| z\right|^{p-2} \times\left(\tilde{X}_{s}(x)-\tilde{X}_{s}(y)\right)^{2} \mid \\
& \quad \leq|p||p-1|\left|\tilde{X}_{s}(x)-\tilde{X}_{s}(y)\right|^{p} .
\end{aligned}
$$

Therefore

$$
\tilde{J}_{t}^{1} \leq|p||p-1| \int_{u}^{t}\left|\tilde{X}_{s}(x)-\tilde{X}_{s}(y)\right|^{p} d s \int_{u}^{t}\left(-\frac{e^{-\Lambda_{s}}}{1-Z_{s \wedge \tau_{n}}}\right)^{2} d N_{s} d N_{s}
$$

The hypothesis 2 is always assumed, so

$$
\tilde{J}_{t}^{1} \leq|p||p-1| Q_{t}^{2} \int_{u}^{t}\left|\tilde{X}_{s}(x)-\tilde{X}_{s}(y)\right|^{p} d s
$$

For $\tilde{J}_{t}^{2}$ we have

$$
\begin{aligned}
& \left|\frac{\partial^{2} f}{\partial z^{2}}\left(\tilde{X}_{s}^{u}(x)-\tilde{X}_{s}^{u}(y)\right) \times\left(\tilde{V}\left(\tilde{X}_{s}^{x}\right)-\tilde{V}\left(\tilde{X}_{s}^{y}\right)\right)^{2}\right| \\
& \quad \leq\left.|p(p-1)| z\right|^{p-2} \tilde{L}^{2}\left(\tilde{X}_{s}^{u}(x)-\tilde{X}_{s}^{u}(y)\right)^{2} \mid \\
& \quad \leq|p||p-1| \tilde{L}^{2}\left|\tilde{X}_{s}(x)-\tilde{X}_{s}(y)\right|^{p} .
\end{aligned}
$$

So

$$
\tilde{J}_{t}^{2} \leq|p \| p-1| \tilde{L}^{2} \int_{u}^{t}\left|\tilde{X}_{s}(x)-\tilde{X}_{s}(y)\right|^{p} d s .
$$

For $\tilde{J}_{t}^{3}$ we have

$$
\begin{aligned}
& \left|\frac{\partial^{2} f}{\partial z^{2}}\left(\tilde{X}_{s}^{u}(x)-\tilde{X}_{s}^{u}(y)\right) \times\left(\tilde{X}_{s}(x)-\tilde{X}_{s}(y)\right)\left(\tilde{V}\left(\tilde{X}_{s}^{x}\right)-\tilde{V}\left(\tilde{X}_{s}^{y}\right)\right)\right| \\
& \quad \leq\left|p(p-1) z^{p-2}\left(\tilde{X}_{s}(x)-\tilde{X}_{s}(y)\right) \tilde{L}\left(\tilde{X}_{s}(x)-\tilde{X}_{s}(y)\right)\right|
\end{aligned}
$$




$$
\leq\left|p(p-1) \tilde{L}\left(\tilde{X}_{s}(x)-\tilde{X}_{s}(y)\right)^{p}\right|
$$

The hypothesis 2 is always assumed, so

$$
\tilde{J}_{t}^{3} \leq 2|p||p-1| \tilde{L} Q_{t} \int_{u}^{t}\left|\tilde{X}_{s}(x)-\tilde{X}_{s}(y)\right|^{p} d s .
$$

Therefore

$$
\begin{aligned}
\tilde{J}_{t}= & \frac{1}{2}\left[\tilde{J}_{t}^{1}+\tilde{J}_{t}^{2}+\tilde{J}_{t}^{3}\right] \\
\tilde{J}_{t}= & \frac{1}{2}\left[|p||p-1| Q_{t}^{2} \int_{u}^{t}\left|\tilde{X}_{s}(x)-\tilde{X}_{s}(y)\right|^{p} d s+|p||p-1| \tilde{L}^{2} \int_{u}^{t}\left|\tilde{X}_{s}(x)-\tilde{X}_{s}(y)\right|^{p} d s\right. \\
& \left.+2|p||p-1| \tilde{L} Q_{t} \int_{u}^{t}\left|\tilde{X}_{s}(x)-\tilde{X}_{s}(y)\right|^{p} d s\right] \\
\tilde{J}_{t} \leq & \frac{1}{2}|p||p-1|\left(Q_{t}+\tilde{L}\right)^{2} \int_{u}^{t}\left|\tilde{X}_{s}(x)-\tilde{X}_{s}(y)\right|^{p} d s .
\end{aligned}
$$

Therefore

$$
\left|\mathbb{E} \tilde{J}_{t \wedge \sigma_{\tilde{\varepsilon}}}\right| \leq \frac{1}{2}|p||p-1|\left(Q_{t}+\tilde{L}\right)^{2} \int_{u}^{t} \mathbb{E}\left|\tilde{X}_{s}(x)-\tilde{X}_{s}(y)\right|^{p} d s .
$$

Summing up these two inequalities 3.1 and 3.2, we obtain

$$
\mathbb{E}\left|\tilde{X}_{t \wedge \sigma_{\tilde{\varepsilon}}}^{u}(x)-\tilde{X}_{t \wedge \sigma_{\tilde{\varepsilon}}}^{u}(y)\right|^{p} \leq|x-y|^{p}+\tilde{C}_{p} \int_{u}^{t} \mathbb{E}\left|\tilde{X}_{s}(x)-\tilde{X}_{s}(y)\right|^{p} d s
$$

where $\tilde{C}_{p}$ is a positive constant.

By Gronwall's inquality, we have:

$$
\mathbb{E}\left|\tilde{X}_{t \wedge \sigma_{\tilde{\varepsilon}}}^{u}(x)-\tilde{X}_{t \wedge \sigma_{\tilde{\varepsilon}}}^{u}(y)\right|^{p} \leq \tilde{K}_{p, u}^{(2)}|x-y|^{p}, \quad u \leq t \leq \infty
$$

such that

$$
\tilde{K}_{p, u}^{(2)}|x-y|^{p}=\exp \left(\tilde{C}_{p} u\right)
$$

Letting $\varepsilon$ tend to 0 , we have:

$$
\mathbb{E}\left|\tilde{X}_{t \wedge \sigma}^{u}(x)-\tilde{X}_{t \wedge \sigma}^{u}(y)\right|^{p} \leq \tilde{K}_{p, u}^{(2)}|x-y|^{p}
$$

where $\sigma$ is the first time such that $\tilde{X}_{t}^{u}(x)=\tilde{X}_{t}^{u}(y)$. However it holds $\sigma=\infty$ 
a.s, since otherwise the left hand side would be infinity if $p<0$. The proof is complete.

The above lemma shows that if $x \neq y$ then $\tilde{X}_{t}^{u}(x) \neq \tilde{X}_{t}^{u}(y)$ holds for all $t$ a.s. But it does not conclude that $\tilde{X}_{t}(., \omega)$ is one to one, since the exceptional null set $\tilde{N}_{x, y}=\left\{\omega / \tilde{X}_{t}^{u}(x)=\tilde{X}_{t}^{u}(y)\right.$ for somet $\}$ depends on the pair $(x, y)$. To overcome this point, we shall apply the Lemma 2 .

In this case we have:

$$
\begin{aligned}
& \tilde{X}_{t}^{u}(x)=x+\int_{u}^{t} \tilde{X}_{s}\left(-\frac{e^{-\Lambda_{s}}}{1-Z_{s \wedge \tau_{n}}}\right) d N_{s}+\int_{u}^{t} \tilde{X}_{s} f\left(\tilde{X}_{s}-\left(1-Z_{s}\right)\right) d Y_{s} \\
& \tilde{X}_{\hat{t}}^{u}(\dot{x})=\dot{x}+\int_{u}^{t} \tilde{X}_{s}\left(-\frac{e^{-\Lambda_{s}}}{1-Z_{s \wedge \tau_{n}}}\right) d N_{s}+\int_{u}^{t} \tilde{X}_{s} f\left(\tilde{X}_{s}-\left(1-Z_{s}\right)\right) d Y_{s} \\
& \tilde{X}_{t}^{u}(y)=y+\int_{u}^{t} \tilde{X}_{s}\left(-\frac{e^{-\Lambda_{s}}}{1-Z_{s \wedge \tau_{n}}}\right) d N_{s}+\int_{u}^{t} \tilde{X}_{s} f\left(\tilde{X}_{s}-\left(1-Z_{s}\right)\right) d Y_{s} \\
& \tilde{X}_{\hat{t}}^{u}(\dot{y})=\dot{y}+\int_{u}^{t} \tilde{X}_{s}\left(-\frac{e^{-\Lambda_{s}}}{1-Z_{s \wedge \tau_{n}}}\right) d N_{s}+\int_{u}^{t} \tilde{X}_{s} f\left(\tilde{X}_{s}-\left(1-Z_{s}\right)\right) d Y_{s} .
\end{aligned}
$$

Putting

$$
\begin{aligned}
\tilde{\eta}_{t}(x, y) & =\frac{1}{\left|\tilde{X}_{t}^{u}(x)-\tilde{X}_{t}^{u}(y)\right|} \\
\tilde{\eta}_{t^{\prime}}\left(x^{\prime}, y^{\prime}\right) & =\frac{1}{\left|\tilde{X}_{t^{\prime}}^{u}\left(x^{\prime}\right)-\tilde{X}_{t^{\prime}}^{u}\left(y^{\prime}\right)\right|} .
\end{aligned}
$$

So

$$
\begin{aligned}
\left|\tilde{\eta}_{t}(x, y)-\tilde{\eta}_{t^{\prime}}\left(x^{\prime}, y^{\prime}\right)\right|^{p} & =\left|\frac{1}{\left|\tilde{X}_{t}^{u}(x)-\tilde{X}_{t}^{u}(y)\right|}-\frac{1}{\left|\tilde{X}_{t^{\prime}}^{u}\left(x^{\prime}\right)-\tilde{X}_{t^{\prime}}^{u}\left(y^{\prime}\right)\right|}\right|^{p} \\
\leq & 2^{p}\left(\frac{1}{\left|\tilde{X}_{t}^{u}(x)-\tilde{X}_{t}^{u}(y)\right|}\right)^{p}\left(\frac{1}{\left|\tilde{X}_{t^{\prime}}^{u}\left(x^{\prime}\right)-\tilde{X}_{t^{\prime}}^{u}\left(y^{\prime}\right)\right|}\right)^{p} \\
& \times\left[\left|\tilde{X}_{t}^{u}(x)-\tilde{X}_{t^{\prime}}^{u}\left(x^{\prime}\right)\right|^{p}+\left|\tilde{X}_{t}^{u}(y)-\tilde{X}_{t^{\prime}}^{u}\left(y^{\prime}\right)\right|^{p}\right] .
\end{aligned}
$$

By Hölder inequality

$$
\begin{aligned}
\mathbb{E}\left|\tilde{\eta}_{t}(x, y)-\tilde{\eta}_{t^{\prime}}\left(x^{\prime}, y^{\prime}\right)\right|^{p} \leq 2^{p}\left(\mathbb{E}\left(\tilde{\eta}_{t}(x, y)^{4 p}\right) \mathbb{E}\left(\tilde{\eta}_{t^{\prime}}\left(x^{\prime}, y^{\prime}\right)^{4 p}\right)\right)^{\frac{1}{4}} \\
\times\left[\left(\mathbb{E}\left|\tilde{X}_{t}^{u}(x)-\tilde{X}_{t^{\prime}}^{u}\left(x^{\prime}\right)\right|^{2 p}\right)^{\frac{1}{2}}+\left(\mathbb{E}\left|\tilde{X}_{t}^{u}(y)-\tilde{X}_{t^{\prime}}^{u}\left(y^{\prime}\right)\right|^{2 p}\right)^{\frac{1}{2}}\right] .
\end{aligned}
$$


By lemme 1 and proposition 1, we have

$$
\begin{aligned}
\mathbb{E}\left|\tilde{\eta}_{t}(x, y)-\tilde{\eta}_{t^{\prime}}\left(x^{\prime}, y^{\prime}\right)\right|^{p} & \leq \tilde{C}_{p, T}|x-y|^{-p}\left|x^{\prime}-y^{\prime}\right|^{-p}\left(\left|x-x^{\prime}\right|^{p}+\left|y-y^{\prime}\right|^{p}+2\left|t-t^{\prime}\right|^{\frac{p}{2}}\right) \\
& \leq \tilde{C}_{p, T} \tilde{\delta}^{-2 p}\left(\left|x-x^{\prime}\right|^{p}+\left|y-y^{\prime}\right|^{p}+2\left|t-t^{\prime}\right|^{\frac{p}{2}}\right)
\end{aligned}
$$

if $|x-y| \geq \tilde{\delta}$ and $\left|x^{\prime}-y^{\prime}\right| \geq \tilde{\delta}$, where $\tilde{C}_{p, T}$ is a positive constant. Then by Kolmogorov Theorem 1, $\tilde{\eta}_{t}(x, y)$ is continuous in $[0, T] \times\{(x, y) /|x-y| \geq \tilde{\delta}\}$. Since $T$ and $\tilde{\delta}$ are arbitrary positive numbers, we get the assertion. The proof is complete.

The above calculus leads immediately the one to one property of the map $\tilde{X}_{t}^{u}(., \omega)$ for all t a.s. We shall next consider the onto property.

\subsection{Proof of the onto property}

In this part we will apply the lemmas 3, 4, and 5 to our model.

Let $T>0$ and $p$ any real number:

$$
\begin{aligned}
\tilde{X}_{t}^{u}(x) & =x+\int_{u}^{t} \tilde{X}_{s}\left(-\frac{e^{-\Lambda_{s}}}{1-Z_{s \wedge \tau_{n}}}\right) d N_{s}+\int_{u}^{t} \tilde{X}_{s} f\left(\tilde{X}_{s}-\left(1-Z_{s}\right)\right) d Y_{s} \\
d \tilde{X}_{t}^{u}(x) & =\tilde{X}_{t}\left(-\frac{e^{-\Lambda_{t}}}{1-Z_{t \wedge \tau_{n}}} d N_{t}+f\left(\tilde{X}_{t}-\left(1-Z_{t}\right)\right) d Y_{t}\right) .
\end{aligned}
$$

We shall apply Itô's formula to the function $f(z)=\left(1+|z|^{2}\right)^{p}$. It holds

$$
\begin{aligned}
& f\left(\tilde{X}_{t}^{u}(x)\right)-f(x) \\
& =\int_{u}^{t} \frac{\partial f}{\partial z}\left(\tilde{X}_{s}^{u}(x)\right)\left[\tilde{X}_{s}(x)\left(-\frac{e^{-\Lambda_{s}}}{1-Z_{s \wedge \tau_{n}}}\right) d N_{s}+\tilde{X}_{s}(x) f\left(\tilde{X}_{s}(x)-\left(1-Z_{s}\right)\right) d Y_{s}\right] \\
& +\frac{1}{2} \int_{u}^{t} \frac{\partial^{2} f}{\partial z^{2}}\left(\tilde{X}_{s}^{u}(x)\right)\left[\tilde{X}_{s}(x)\left(-\frac{e^{-\Lambda_{s}}}{1-Z_{s \wedge \tau_{n}}}\right) d N_{s}+\tilde{X}_{s}(x) f\left(\tilde{X}_{s}(x)-\left(1-Z_{s}\right)\right) d Y_{s}\right]^{2} \\
& f\left(\tilde{X}_{t}^{u}(x)\right)-f(x)=\tilde{I}_{t}+\tilde{J}_{t} \text { such that } \\
& \quad \tilde{I}_{t}=\int_{u}^{t} \frac{\partial f}{\partial z}\left(\tilde{X}_{s}^{u}(x)\right) \\
& \quad \times\left[\tilde{X}_{s}(x)\left(-\frac{e^{-\Lambda_{s}}}{1-Z_{s \wedge \tau_{n}}}\right) d N_{s}+\tilde{X}_{s}(x) f\left(\tilde{X}_{s}(x)-\left(1-Z_{s}\right)\right) d Y_{s}\right]
\end{aligned}
$$




$$
\begin{aligned}
\tilde{J}_{t}= & \frac{1}{2} \int_{u}^{t} \frac{\partial^{2} f}{\partial z^{2}}\left(\tilde{X}_{s}^{u}(x)\right) \\
& \times\left[\tilde{X}_{s}(x)\left(-\frac{e^{-\Lambda_{s}}}{1-Z_{s \wedge \tau_{n}}}\right) d N_{s}+\tilde{X}_{s}(x) f\left(\tilde{X}_{s}(x)-\left(1-Z_{s}\right)\right) d Y_{s}\right]^{2} .
\end{aligned}
$$

For $\tilde{I}_{t}$, we have

$$
\begin{aligned}
\tilde{I}_{t}= & \int_{u}^{t} \frac{\partial f}{\partial z}\left(\tilde{X}_{s}^{u}(x)\right) \\
& \times\left[\tilde{X}_{s}(x)\left(-\frac{e^{-\Lambda_{s}}}{1-Z_{s \wedge \tau_{n}}}\right) d N_{s}+\tilde{X}_{s}(x) f\left(\tilde{X}_{s}(x)-\left(1-Z_{s}\right)\right) d Y_{s}\right] \\
\tilde{I}_{t}= & \int_{u}^{t} \frac{\partial f}{\partial z}\left(\tilde{X}_{s}^{u}(x)\right) \tilde{X}_{s}(x)\left(-\frac{e^{-\Lambda_{s}}}{1-Z_{s \wedge \tau_{n}}}\right) d N_{s} \\
& +\int_{u}^{t} \frac{\partial f}{\partial z}\left(\tilde{X}_{s}^{u}(x)\right) \tilde{X}_{s}(x) f\left(\tilde{X}_{s}(x)-\left(1-Z_{s}\right)\right) d Y_{s}
\end{aligned}
$$

$=\tilde{I}_{t}^{1}+\tilde{I}_{t}^{2}$ such that

$$
\begin{aligned}
& \tilde{I}_{t}^{1}=\int_{u}^{t} \frac{\partial f}{\partial z}\left(\tilde{X}_{s}^{u}(x)\right) \tilde{X}_{s}(x)\left(-\frac{e^{-\Lambda_{s}}}{1-Z_{s \wedge \tau_{n}}}\right) d N_{s} \\
& \tilde{I}_{t}^{2}=\int_{u}^{t} \frac{\partial f}{\partial z}\left(\tilde{X}_{s}^{u}(x)\right) \tilde{X}_{s}(x) f\left(\tilde{X}_{s}(x)-\left(1-Z_{s}\right)\right) d Y_{s} .
\end{aligned}
$$

For $\tilde{I}_{t}^{1}$, note $\frac{\partial f}{\partial z}=2 p z\left(1+|z|^{2}\right)^{p-1}$ and the hypothesis 2 is always assumed,

so

$$
\begin{gathered}
\tilde{I}_{t}^{1}=\int_{u}^{t} \frac{\partial f}{\partial z}\left(\tilde{X}_{s}^{u}(x)\right) \tilde{X}_{s}(x)\left(-\frac{e^{-\Lambda_{s}}}{1-Z_{s \wedge \tau_{n}}}\right) d N_{s} \\
\left|\frac{\partial f}{\partial z}\left(\tilde{X}_{s}^{u}(x)\right) \tilde{X}_{s}(x)\right| \\
\leq 2|p||z|\left(1+|z|^{2}\right)^{p-1}\left|\tilde{X}_{s}(x)\right| \\
\leq 2|p|\left(1+\left|\tilde{X}_{s}(x)\right|^{2}\right)^{p} .
\end{gathered}
$$

Therefore

$$
\tilde{I}_{t}^{1} \leq 2|p| Q_{t} \int_{u}^{t}\left(1+\left|\tilde{X}_{s}(x)\right|^{2}\right)^{p} d s
$$


For $\tilde{I}_{t}^{2}$, we have

$$
\tilde{I}_{t}^{2}=\int_{u}^{t} \frac{\partial f}{\partial z}\left(\tilde{X}_{s}^{u}(x)\right) \tilde{X}_{s}(x) f\left(\tilde{X}_{s}(x)-\left(1-Z_{s}\right)\right) d Y_{s} .
$$

Noting

$$
\tilde{V}\left(\tilde{X}_{s}^{x}\right)=\tilde{X}_{s}(x) f\left(\tilde{X}_{s}(x)-\left(1-Z_{s}\right)\right) .
$$

Let $\tilde{K}$ be a positive constant such that

$$
\begin{aligned}
V\left(\tilde{X}_{s}^{x}\right) & \leq \tilde{K}\left(1+\left|\tilde{X}_{s}(x)\right|^{2}\right)^{\frac{1}{2}} \\
\left|\frac{\partial f}{\partial z}\left(\tilde{X}_{s}^{u}(x)\right) \tilde{X}_{s}(x) \times \tilde{V}\left(\tilde{X}_{s}^{x}\right)\right| & \leq 2|p||z|\left(1+|z|^{2}\right)^{p-1} \tilde{K}\left(1+\left|\tilde{X}_{s}(x)\right|^{2}\right)^{\frac{1}{2}} \\
& \leq 2|p| \tilde{K}\left(1+\left|\tilde{X}_{s}(x)\right|^{2}\right)^{p} .
\end{aligned}
$$

So

$$
\tilde{I}_{t}^{2} \leq 2|p| \tilde{K} \int_{u}^{t}\left(1+\left|\tilde{X}_{s}(x)\right|^{2}\right)^{p} d s .
$$

Therefore

$$
\begin{aligned}
\tilde{I}_{t} & \leq 2|p| Q_{t} \int_{u}^{t}\left(1+\left|\tilde{X}_{s}(x)\right|^{2}\right)^{p} d s+2|p| \tilde{K} \int_{u}^{t}\left(1+\left|\tilde{X}_{s}(x)\right|^{2}\right)^{p} d s \\
& \leq 2|p|\left(Q_{t}+\tilde{K}\right) \int_{u}^{t}\left(1+\left|\tilde{X}_{s}(x)\right|^{2}\right)^{p} d s .
\end{aligned}
$$

We have

$$
\left|\mathbb{E} \tilde{I}_{t}\right| \leq 2|p|\left(Q_{t}+\tilde{K}\right) \int_{u}^{t} \mathbb{E}\left(1+\left|\tilde{X}_{s}(x)\right|^{2}\right)^{p} d s
$$

Next, for $\tilde{J}_{t}$ we have

$$
\begin{aligned}
\tilde{J}_{t}= & \frac{1}{2} \int_{u}^{t} \frac{\partial^{2} f}{\partial z^{2}}\left(\tilde{X}_{s}^{u}(x)\right) \times\left[\tilde{X}_{s}(x)\left(-\frac{e^{-\Lambda_{s}}}{1-Z_{s \wedge \tau_{n}}}\right) d N_{s}\right. \\
& \left.+\tilde{X}_{s}(x) f\left(\tilde{X}_{s}(x)-\left(1-Z_{s}\right)\right) d Y_{s}\right]^{2} \\
\tilde{J}_{t}= & \frac{1}{2} \int_{u}^{t} \frac{\partial^{2} f}{\partial z^{2}}\left(\tilde{X}_{s}^{u}(x)\right)\left[\tilde{X}_{s}(x)^{2}\left(-\frac{e^{-\Lambda_{s}}}{1-Z_{s \wedge \tau_{n}}}\right)^{2} d N_{s} d N_{s}+\tilde{V}\left(\tilde{X}_{s}^{x}\right)^{2} d Y_{s} d Y_{s}\right.
\end{aligned}
$$




$$
\left.+2 \tilde{X}_{s}^{u}(x)\left(-\frac{e^{-\Lambda_{s}}}{1-Z_{s \wedge \tau_{n}}}\right) \tilde{V}\left(\tilde{X}_{s}^{x}\right) d N_{s} d Y_{s}\right]
$$

Noting $\tilde{J}_{t}=\frac{1}{2}\left[\tilde{J}_{t}^{1}+\tilde{J}_{t}^{2}+\tilde{J}_{t}^{3}\right]$, such that

$$
\begin{aligned}
& \tilde{J}_{t}^{1}=\int_{u}^{t} \frac{\partial^{2} f}{\partial z^{2}}\left(\tilde{X}_{s}^{u}(x)\right) \times \tilde{X}_{s}(x)^{2}\left(-\frac{e^{-\Lambda_{s}}}{1-Z_{s \wedge \tau_{n}}}\right)^{2} d N_{s} d N_{s} \\
& \tilde{J}_{t}^{2}=\int_{u}^{t} \frac{\partial^{2} f}{\partial z^{2}}\left(\tilde{X}_{s}^{u}(x)\right) \times \tilde{V}\left(\tilde{X}_{s}^{x}\right)^{2} d Y_{s} d Y_{s} \\
& \tilde{J}_{t}^{3}=2 \int_{u}^{t} \frac{\partial^{2} f}{\partial z^{2}}\left(\tilde{X}_{s}^{u}(x)\right) \times \tilde{X}_{s}^{u}(x)\left(-\frac{e^{-\Lambda_{s}}}{1-Z_{s \wedge \tau_{n}}}\right) \tilde{V}\left(\tilde{X}_{s}^{x}\right) d N_{s} d Y_{s}
\end{aligned}
$$

and note that

$$
\frac{\partial^{2} f}{\partial z^{2}}=2 p\left(1+|z|^{2}\right)^{p-1}+4 p(p-1) z^{2}\left(1+|z|^{2}\right)^{p-2}
$$

Then for $\tilde{J}_{t}^{1}$ we have

$$
\begin{aligned}
& \tilde{J}_{t}^{1}=\int_{u}^{t} \frac{\partial^{2} f}{\partial z^{2}}\left(\tilde{X}_{s}^{u}(x)\right) \times \tilde{X}_{s}(x)^{2}\left(-\frac{e^{-\Lambda_{s}}}{1-Z_{s \wedge \tau_{n}}}\right)^{2} d N_{s} d N_{s} \\
&\left|\frac{\partial^{2} f}{\partial z^{2}}\left(\tilde{X}_{s}^{u}(x)\right) \times \tilde{X}_{s}(x)^{2}\right| \\
& \quad \leq\left|\left(2 p\left(1+|z|^{2}\right)^{p-1}+4 p(p-1) z^{2}\left(1+|z|^{2}\right)^{p-2}\right) \tilde{X}_{s}(x)^{2}\right| \\
& \quad \leq 2|p|(2(p-1)+1)\left(1+\left|\tilde{X}_{s}(x)\right|^{2}\right)^{p} .
\end{aligned}
$$

Therefore

$\tilde{J}_{t}^{1} \leq 2|p|(2(p-1)+1) \int_{u}^{t}\left(1+\left|\tilde{X}_{s}(x)\right|^{2}\right)^{p} d s \int_{u}^{t}\left(-\frac{e^{-\Lambda_{s}}}{1-Z_{s \wedge \tau_{n}}}\right)^{2} d N_{s} d N_{s}$.

By hypothesis 2, we have

$$
\tilde{J}_{t}^{1} \leq 2|p|(2(p-1)+1) Q_{t}^{2} \int_{u}^{t}\left(1+\left|\tilde{X}_{s}(x)\right|^{2}\right)^{p} d s .
$$


For $\tilde{J}_{t}^{2}$, we have

$$
\begin{gathered}
\tilde{J}_{t}^{2}=\int_{u}^{t} \frac{\partial^{2} f}{\partial z^{2}}\left(\tilde{X}_{s}^{u}(x)\right) \times \tilde{V}\left(\tilde{X}_{s}^{x}\right)^{2} d Y_{s} d Y_{s} \\
\left|\frac{\partial^{2} f}{\partial z^{2}}\left(\tilde{X}_{s}^{u}(x)\right) \times \tilde{V}\left(\tilde{X}_{s}^{x}\right)^{2}\right| \\
\leq\left|\left(2 p\left(1+|z|^{2}\right)^{p-1}+4 p(p-1) z^{2}\left(1+|z|^{2}\right)^{p-2}\right) \times \tilde{K}^{2}\left(1+\left|\tilde{X}_{s}(x)\right|^{2}\right)\right| \\
\leq 2|p|(2(p-1)+1) \tilde{K}^{2}\left(1+\left|\tilde{X}_{s}(x)\right|^{2}\right)^{p} .
\end{gathered}
$$

Therefore

$$
\tilde{J}_{t}^{2} \leq 2|p|(2(p-1)+1) \tilde{K}^{2} \int_{u}^{t}\left(1+\left|\tilde{X}_{s}(x)\right|^{2}\right)^{p} d s
$$

For $\tilde{J}_{t}^{3}$, we have

$$
\begin{aligned}
\tilde{J}_{t}^{3} & =2 \int_{u}^{t} \frac{\partial^{2} f}{\partial z^{2}}\left(\tilde{X}_{s}^{u}(x)\right) \times \tilde{X}_{s}(x)\left(-\frac{e^{-\Lambda_{s}}}{1-Z_{s \wedge \tau_{n}}}\right) \tilde{V}\left(\tilde{X}_{s}^{x}\right) d N_{s} d Y_{s} \\
& \left|\frac{\partial^{2} f}{\partial z^{2}}\left(\tilde{X}_{s}^{u}(x)\right) \times \tilde{X}_{s}(x) \tilde{V}\left(\tilde{X}_{s}^{x}\right)\right| \\
& \leq\left|\left(2 p\left(1+|z|^{2}\right)^{p-1}+4 p(p-1) z^{2}\left(1+|z|^{2}\right)^{p-2}\right) \tilde{K}^{2}\left(1+\left|\tilde{X}_{s}(x)\right|^{2}\right)^{\frac{1}{2}} \tilde{X}_{s}(x)\right| \\
& \leq 2|p|(2(p-1)+1) \tilde{K}\left(1+\left|\tilde{X}_{s}(x)\right|^{2}\right)^{p} .
\end{aligned}
$$

The hypothesis 2 is always assumed, so

$$
\tilde{J}_{t}^{3} \leq 4|p|(2(p-1)+1) \tilde{K} Q_{t} \int_{u}^{t}\left(1+\left|\tilde{X}_{s}(x)\right|^{2}\right)^{p} d s
$$

Therefore

$$
\begin{aligned}
\tilde{J}_{t}= & \frac{1}{2}\left[\tilde{J}_{t}^{1}+\tilde{J}_{t}^{2}+\tilde{J}_{t}^{3}\right] \\
\tilde{J}_{t}= & \frac{1}{2}\left[2|p|(2(p-1)+1) Q_{t}^{2} \int_{u}^{t}\left(1+\left|\tilde{X}_{s}(x)\right|^{2}\right)^{p} d s\right. \\
& +2|p|(2(p-1)+1) \tilde{K}^{2} \times \int_{u}^{t}\left(1+\left|\tilde{X}_{s}(x)\right|^{2}\right)^{p} d s
\end{aligned}
$$




$$
\begin{array}{r}
\left.+4|p|(2(p-1)+1) \tilde{K} Q_{t} \int_{u}^{t}\left(1+\left|\tilde{X}_{s}(x)\right|^{2}\right)^{p} d s\right] \\
\tilde{J}_{t} \leq|p|(2(p-1)+1)\left(Q_{t}+\tilde{K}\right)^{2} \int_{u}^{t}\left(1+\left|\tilde{X}_{s}(x)\right|^{2}\right)^{p} d s .
\end{array}
$$

So

$$
\left|\mathbb{E} \tilde{J}_{t}\right| \leq|p|(2(p-1)+1)\left(Q_{t}+\tilde{K}\right)^{2} \int_{u}^{t} \mathbb{E}\left(1+\left|\tilde{X}_{s}(x)\right|^{2}\right)^{p} d s .
$$

Summing up these two inequalities 3.3 and 3.4 , we obtain

$$
\mathbb{E}\left(1+\left|\tilde{X}_{s}(x)\right|^{2}\right)^{p} \leq\left(1+|x|^{2}\right)^{p}+\text { const } \times \int_{u}^{t} \mathbb{E}\left(1+\left|\tilde{X}_{s}(x)\right|^{2}\right)^{p} d s
$$

By Gronwall's inequality, we have

$$
\mathbb{E}\left(1+\left|\tilde{X}_{s}(x)\right|^{2}\right)^{p} \leq\left(1+|x|^{2}\right)^{p} \times \exp \left(\tilde{C}_{p, u}\right)
$$

such that

$$
\tilde{C}_{p, u}=\text { const } \times \int_{u}^{t} \mathbb{E}\left(1+\left|\tilde{X}_{s}(x)\right|^{2}\right)^{p} d s
$$

and

$$
\tilde{K}_{p, u}^{3}=\exp \left(\tilde{C}_{p, u}\right)
$$

So, we have the inequality of the Lemma 3

$$
\mathbb{E}\left(1+\left|\tilde{X}_{s}(x)\right|^{2}\right)^{p} \leq \tilde{K}_{p, u}^{3}\left(1+|x|^{2}\right)^{p}
$$

Now, taking negative $p$ in the above calculus, we see that $\left|\tilde{X}_{t}(x)\right|$ tends to infinity in probability as $x$ tends sequencially to infinity. We shall prove a stronger convergence.

Let $\overline{\mathbb{R}}=\mathbb{R} \cup\{\infty\}$ be the one point compactification of $\mathbb{R}$. Set

$$
\begin{aligned}
& \tilde{X}_{t}^{u}(x)=x+\int_{u}^{t} \tilde{X}_{s}\left(-\frac{e^{-\Lambda_{s}}}{1-Z_{s \wedge \tau_{n}}}\right) d N_{s}+\int_{u}^{t} \tilde{X}_{s} f\left(\tilde{X}_{s}-\left(1-Z_{s}\right)\right) d Y_{s} \\
& \tilde{\eta}_{t}(x)= \begin{cases}\frac{1}{1+\left|\tilde{X}_{t}(x)\right|}, & \text { if } x \in \mathbb{R}, \\
0, & \text { if } x=\infty .\end{cases}
\end{aligned}
$$


Evidently $\tilde{\eta}_{t}(x)$ is continuous in $[0, \infty) \times \mathbb{R}$. Thus just to prove the continuity in the vicinity of infinity. Suppose $p>2$. It holds

$$
\left|\tilde{\eta}_{t}(x)-\tilde{\eta}_{s}(y)\right|^{p} \leq \tilde{\eta}_{t}(x)^{p} \tilde{\eta}_{s}(y)^{p}\left|\tilde{X}_{t}(x)-\tilde{X}_{s}(y)\right|^{p}
$$

By Hölder inequality, proposition 1] and Lemma 3, we have

$$
\begin{aligned}
\mathbb{E}\left|\tilde{\eta}_{t}(x)-\tilde{\eta}_{s}(y)\right|^{p} & \leq\left(\mathbb{E} \tilde{\eta}_{t}(x)^{4 p}\right)^{\frac{1}{4}}\left(\mathbb{E} \tilde{\eta}_{s}(y)^{4 p}\right)^{\frac{1}{4}}\left(\mathbb{E}\left|\tilde{X}_{t}(x)-\tilde{X}_{s}(y)\right|^{2 p}\right)^{\frac{1}{2}} \\
& \leq \tilde{C}_{p, T}(1+|x|)^{-p}(1+|y|)^{-p}\left(|x-y|^{p}+|t-s|^{\frac{p}{2}}\right)
\end{aligned}
$$

if $t, s \in[0, T]$ and $x, y \in \mathbb{R}$, where $\tilde{C}_{p, T}$ is a positive constant. Set

$$
\frac{1}{x}=x^{-1}
$$

Since

$$
\frac{|x-y|}{(1+|x|)(1+|y|)} \leq\left|\frac{1}{x}-\frac{1}{y}\right| .
$$

We get the inequality

$$
\mathbb{E}\left|\tilde{\eta}_{t}(x)-\tilde{\eta}_{s}(y)\right|^{p} \leq \tilde{C}_{p, T}\left(\left|\frac{1}{x}-\frac{1}{y}\right|^{p}+|t-s|^{\frac{p}{2}}\right) .
$$

Define

$$
\bar{\eta}_{t}(x)= \begin{cases}\tilde{\eta}_{t}\left(\frac{1}{x}\right), & \text { if } x \neq 0 \\ 0, & \text { if } x=0\end{cases}
$$

Then the above inequality implies

$$
\mathbb{E}\left|\bar{\eta}_{t}(x)-\bar{\eta}_{s}(y)\right|^{p} \leq \tilde{C}_{p, T}\left(|x-y|^{p}+|t-s|^{\frac{p}{2}}\right), \quad x \neq 0, y \neq 0 .
$$

In case $y=0$, we have

$$
\mathbb{E}\left|\bar{\eta}_{t}(x)\right|^{p} \leq \tilde{C}_{p, T}|x|^{p}
$$

Therefore $\bar{\eta}_{t}(x)$ is continuous in $[0, \infty) \times \mathbb{R}$ by Kolmogorov's theorem. This proves that $\tilde{\eta}_{t}(x)$ is continuous in $[0, \infty) \times$ neighborhood of infinity.

So, define a stochastic process $\bar{X}_{t}$ on $\overline{\mathbb{R}}=\mathbb{R} \cup\{\infty\}$ by

$$
\bar{X}_{t}(x)= \begin{cases}\tilde{X}_{t}(x), & \text { if } x \in \mathbb{R} \\ \infty, & \text { if } x=\infty\end{cases}
$$


Then $\bar{X}_{t}(x)$ is continuous sur $[0, \infty) \times \mathbb{R}$ by the previous lemma. Thus for each $t>0$, the map $\bar{X}_{t}(., \omega)$ is homotopic to the identity map on $\overline{\mathbb{R}}$. Then $\bar{X}_{t}(., \omega)$ is an onto map of $\overline{\mathbb{R}}$ by a well known homotopic theory. Now, the map $\bar{X}_{t}$ is a homeomorphism of $\overline{\mathbb{R}}$, since it is one to one, onto and continuous. Since $\infty$ is the invariant point of the map $\bar{X}_{t}$, we see that $\tilde{X}_{t}$ is a homeomorphism of $\mathbb{R}$. This completes the proof of Theorem 1 .

\section{Conclusion}

This work contains a new and original methodological approach to the subject in question and could therefore be a good contribution to the theory of stochastic differential equations, based on a very interesting lemmas of Kunita [2]. Some difficulties have been encountered because the subject deals with a difficult area "Stochastic differential geometry". As prospects, we try to prove the same result of the paper, but on manifolds.

\section{References}

1. Monique Jeanblanc and Shiqi Song, Random times with given survival probability and their F-martingale decomposition formula, Stochastic Processes And their Applications, 121(2010-2011).

2. H. Kunita, On the decomposition of solutions of stochastic differential equations, Proceeding of the LMS Symposium on Stoch. Diff. Eqs. Durham, Juillet (1980).

3. K. D. Elworthy, Stochastic Dynamical Systems and Their Flows, stochastic analysis ed. by A.Friedman and M. Pinsky, 79-95, Academic press, New York, 1978.

4. P. Malliavin, Stochastic calculus of variation and hypoelliptic operators, Kyoto, Conference, 1976, Wiley 1978, 195-263.

5. N. Ikeda and S. Watanabe, Stochastic differential equations and diffusion processes, forthcoming book.

6. J. M. Bismut, Flots stochastiques et formula de Ito-Stratonovich généralisée, $C . R$. Acad. Sci. Paris, t. 290 (10 mars 1980).

7. F. Benziadi and A. Kandouci, The application of Kolmogorov's theorem in the onedefault model, Journal of Mathematical Sciences and Applications E-Notes, 4(2016), , No.2, 71-78.

8. D. W. Stroock and S. R. S. Varadhan, Multidimensional Diffusion Processes, SpringerVerlag, New York, 1979.

9. Y. Ogura and T. Yamada, On the strong comparison theorem of solutions of stochastic differential equations, to appear. 